\title{
La montée de la préoccupation scolaire des familles populaires
}

Le cas de la Suisse romande

The increasing concern over schooling among working-class families. The case of French-speaking Switzerland

La subida de la preocupación escolar de las familias populares. El caso de la Suiza francófona

\section{Christophe Delay}

\section{(2) OpenEdition}

Journals

Édition électronique

URL : https://journals.openedition.org/ries/3149

DOI : $10.4000 /$ ries.3149

ISSN : 2261-4265

Éditeur

France Education international

Édition imprimée

Date de publication : 1 avril 2013

Pagination : 125-133

ISBN : 978-2-85420-599-2

ISSN : $1254-4590$

Référence électronique

Christophe Delay, «La montée de la préoccupation scolaire des familles populaires », Revue internationale d'éducation de Sèvres [En ligne], 62 I avril 2013, mis en ligne le 01 avril 2015, consulté le 06 juillet 2021. URL : http://journals.openedition.org/ries/3149 ; DOI : https://doi.org/10.4000/ries. 3149 


\section{La montée de la préoccupation scolaire des familles populaires}

\section{Le cas de la Suisse Romande}

\section{Christophe Delay}

La majorité des pays occidentaux ont connu, depuis les années 1960, un phénomène de massification scolaire croissant, avec l'instauration tout d'abord de l'école secondaire obligatoire pour tous les élèves, quel que soit leur milieu social, puis la généralisation des formations secondaires post-obligatoires, ce qui a eu pour conséquence un allongement progressif des scolarités juvéniles ainsi qu'une intensification de la préoccupation scolaire au sein des familles. Alors que cette question a été largement débattue en France, elle a été négligée ces dernières années en Suisse, du moins sa partie romande : les travaux portant sur le rapport à l'école des familles (Montandon, 1991) n’ont pas été renouvelés depuis les années 1980.

La présente contribution souhaite mieux comprendre le rapport à l'école des familles populaires suisses romandes en se focalisant plus particulièrement sur le point suivant: la massification du système d'enseignement secondaire a-t-elle conduit ces familles à intégrer l'école dans leurs stratégies éducatives ? Pour répondre à cette question, nous nous appuyons sur une enquête ${ }^{1}$ réalisée à Genève en 2006-2007 dans un établissement scolaire primaire appartenant au Réseau d'enseignement prioritaire qui reçoit - étant donné la forte concentration d'élèves de milieux populaires et issus de l'immigration - davantage de ressources monétaires, ceci pour «favoriser l'égalité des chances en matière de réussite scolaire $»^{2}$. Notre présence régulière in situ nous a permis d'observer de nombreux élèves âgés de 4 à 12 ans puis d'accéder, sur la base des registres scolaires enseignants où étaient inscrits la profession des parents, à des familles populaires ${ }^{3}$ auxquelles nous avons envoyé un courrier pour solliciter un entretien. Étant donné la forte surreprésentation des familles étrangères, nous avons fait appel à notre cercle d'interconnaissances (ainsi qu'à celui de collègues) pour accéder à plusieurs familles de nationalité suisse habitant hors du quartier

1. Financée par le Fonds national suisse de la recherche scientifique (2005-2008), elle s'inscrivait dans le projet plus vaste intitulé « Enfants en danger, familles dangereuses : les métamorphoses de la question sociale sous le règne du nouvel esprit du capitalisme » (direction : F. Schultheis).

2. Département de l'instruction publique (2010) Réseau d'enseignement prioritaire, http://www.geneve.ch/ enseignement_primaire/rep/\#pourquoi/

3. Ayant toutes un emploi d'exécution impliquant des situations fortement assujetties, souvent répétitives et associées à des ressources monétaires étroites : soit des ouvriers mais aussi des employés tels que personnels d'entretien, aide aux personnes dépendantes, serveuses, vendeuses ou travaillant dans les secteurs publics (transports publics, télécommunications). 
investigué, en Romandie, dans le but de pouvoir analyser les catégories populaires dans leur ensemble. Au total, nous avons réalisé 44 entretiens avec quarante familles populaires, en majorité faiblement diplômées. Désireux d'éviter toute homogénéisation, nous avons sélectionné des élèves dont les scolarités étaient diverses - bonnes, moins bonnes - ces dernières étant majoritaires dans notre échantillon ${ }^{4}$.

\section{MASSIFICATION DU SYSTÈME D'ENSEIGNEMENT ET MONTÉE DE LA PRÉOCCUPATION SCOLAIRE}

Avant de mettre en évidence quelques aspects du rapport à l'école des familles populaires romandes, il convient de décrire brièvement le processus de massification qu'a connu le système d'enseignement genevois, en s'appuyant sur le travail monographique de l'historien Müller. Depuis sa création en 1872 et jusque dans les années 1960, le système scolaire genevois s'est caractérisé par son caractère tant dual que ségrégatif: l'école primaire publique est suivie par le peuple tandis que l'école secondaire à plein temps est réservée aux notables. Les élèves des classes populaires quittent l'école précocement, à l'âge de 13 ans, pour intégrer rapidement le monde du travail salarié (usine), avec l'accord de leurs parents qui ne considèrent pas l'école comme un moyen d'ascension sociale. Müller (2007) souligne pour 1930 les espoirs faibles d'ascension sociale par l'école au sein des familles populaires, parlant d'aspirations « modestes $»^{5}$. Dans un contexte plus général d'économie en stagnation (1914-1945) les chances individuelles d'accès à un statut social supérieur sont faibles en Suisse. De ce fait, les revendications d'amélioration sont d'abord collectives. "Il s'agit pour les classes populaires, dominantes du point de vue du nombre, d'assurer à la famille, par le travail, une certaine sécurité matérielle. La mobilité sociale ne fait pas vraiment partie de l'agenda" (Sapin et al., 2007).

Les choses vont changer dès 1957, avec tout d'abord l'introduction de la gratuité de toutes les études secondaires, mesure qui incite les familles populaires à faire tenter leur chance dans l'enseignement secondaire aux enfants qui semblent en avoir les capacités scolaires. "Ces évolutions démontrent aussi que l'idée qu'une certaine réussite scolaire est une ressource accessible pour une forme de promotion sociale s'insinue progressivement dans les consciences " (Müller, 2007). Les années 1960 se caractérisent par une croissance économique sans précédent. Face d'une part à la demande en nombre toujours accru de personnel hautement qualifié et possédant une instruction très poussée permettant de soutenir la croissance économique; d'autre part au souci des milieux de

4. On sait en effet que les performances aux examens de fin de scolarité primaire sont, comparativement aux élèves des milieux favorisés, beaucoup plus basses en milieu ouvrier (SRED, 2011).

5. Des constats similaires sont établis en France pour cette époque (Poullaouec, 2010). 
gauche d'offrir à tous les enfants des chances égales à l'école, le système d'enseignement secondaire va se massifier. En 1969 est créé le cycle d'orientation, école unique du secondaire inférieur qui s'ouvre désormais à des élèves venus de milieux sociaux plus modestes et prolonge leur scolarité jusqu'à l'âge de 15 ans. Ce système d'enseignement unifié remplace les diverses anciennes écoles secondaires qui cloisonnaient leur public en fonction de l'origine sociale. Cette lente mutation du système d'enseignement a conduit à une diffusion culturelle d'une représentation nouvelle des usages possibles du système d'enseignement comme moyen d'ascension sociale dans les différentes couches de la population " selon une progression descendante socialement (des classes moyennes aux classes populaires) » (Müller, 2007). Mais un second processus de massification, au niveau secondaire supérieur cette fois, est déjà à l'œuvre dans les années 1960-1970 : la résorption rapide du retard scolaire de masse mise en œuvre par l'institution scolaire (via l'adoucissement de la sélection, nécessaire pour conduire le plus grand nombre à poursuivre des études) ainsi que la pré-scolarisation croissante des enfants, ceci dès la petite enfance, ont des répercussions sur l'allongement de la scolarité après 15 ans. Dans l'enseignement post-obligatoire, les taux de scolarisation à plein temps passent de 28 à $44 \%$ entre 1960 et 1974 . Ce processus s'est prolongé jusqu'à aujourd'hui : la massification de l'enseignement secondaire supérieur est désormais quasiment achevée, les taux d'obtention des diplômes à ce niveau étant en 2009 pour Genève de l'ordre de 93,5\% (SRED, 2011), et autour de $90 \%$ en Suisse, l'un des taux les plus élevés parmi l'ensemble des pays occidentaux (Meyer, 2009). En conséquence, le pourcentage de jeunes suisses envisageant d'entrer directement sur le marché du travail après l'école obligatoire est quasiment nul. On est désormais loin de l'époque des années 1960 où entre 40 et $50 \%$ des élèves quittaient l'école genevoise à l'âge de 15 ans pour entrer directement sur le marché de l'emploi (Hutmacher, 1998). Corollaire de ce vaste processus de massification scolaire, on peut constater à Genève l'allongement de l'espérance scolaire juvénile, toutes classes sociales confondues (de 19,5 ans en moyenne en 1980 à 20,8 en 2009) ${ }^{6}$.

La section qui suit vise à déterminer quel a été l'impact de la massification de l'enseignement secondaire sur les aspirations éducatives des familles populaires contemporaines.

\section{ÉCHAPPER AU DESTIN OUVRIER PAR LA POURSUITE D'UNE FORMATION EN ÉCOLE}

Il ressort de l'enquête que la très forte majorité des parents ont une vision de l'avenir professionnel de leur progéniture en rupture avec la transmission d'un héritage ouvrier. Lorsqu'ils mentionnent les souhaits qu'ils envisagent pour

6. Les chiffres sont quasiment identiques pour la Suisse (Office fédéral de la statistique, 2007). 
leurs enfants, bon nombre de parents récusent leur activité professionnelle au rang de figure repoussoir, souhaitant que ces derniers, lorsqu'ils effectuent des scolarités non entachées d'un redoublement, puissent continuer dans la voie des études et ainsi échapper à la condition ouvrière, marquée par la pénibilité physique, les tâches répétitives, un travail "salissant » et peu "épanouissant ». Les propos du couple De Sousa, ouvriers polisseurs, dont les deux fils réussissent scolairement, sont à ce propos éloquents :

"Je dis: "si vous étudiez tout ça, vous pouvez avoir une vie plus facile, un travail plus propre que le mien", parce que le polissage c'est un travail sale. S'ils arrivent à trouver quelque chose de mieux, ça sera bien pour eux ».

Tout se passe ainsi comme si bon nombre de familles ne vivaient plus leur métier sur le mode de la fierté comme dans les années 1950, où elles s'attendaient à ce que leurs enfants quittent le plus tôt possible l'école pour s'insérer dans les usines et reproduire leur propre position, mais davantage sur le mode de la honte ${ }^{7}$.

Les parents souhaitent désormais que leurs enfants poursuivent une formation après l'école obligatoire, de préférence dans la voie scolaire à plein temps (voir aussi Decarro, 1998), ce qui doit leur permettre d'obtenir des qualifications qu'ils pourront faire valoir sur le marché du travail. Méconnaissant le système de formation genevois, soit parce qu'ils ont migré en Romandie après une scolarité effectuée dans le pays d'origine, soit pour les Suisses du fait de scolarités courtes, ils évoquent la poursuite indifférenciée "d'études », au projet et à l'horizon vagues ( le plus loin» "jusqu’à la fin») devant permettre d'acquérir des diplômes eux-mêmes exprimés de manière floue ( « un bon diplôme » " avoir quelque chose dans les mains »). La formation par apprentissage dual en école et en entreprise, qui conduit ultérieurement à des emplois d'ouvriers/employés, ne constitue pas un "premier choix» parental, pour le moins tant que leurs enfants ne connaissent pas le redoublement. Il intervient en général a posteriori, comme "second choix » à défaut d'une poursuite dans la voie scolaire, lorsque les difficultés scolaires s'accumulent.

"En tout cas une chose dont je suis sûre avec Jean, c'est qu'il fera jamais d'études hein! [rires]... ça c'est clair, il va s'arrêter à l'école obligatoire... peut-être qu'il peut tomber sur un prof qui lui donnerait une autre envie, bon, l'espoir fait vivre. Mais les études, Jean... je pense pas. » $\left[\mathrm{M}^{\mathrm{me}} \mathrm{Hässler,} \mathrm{femme}\right.$ au foyer, un fils étiqueté "élève en grande difficulté »].

La préférence marquée des parents pour une poursuite de leurs enfants dans la voie scolaire doit permettre à ces derniers d'exercer ultérieurement un «bon métier » qu'ils auront eux-mêmes choisi, phénomène nouveau par rapport à un passé récent où les parents déclaraient à propos de leurs enfants : "ils feront ce qu'ils pourront ».

7. En France, voir Beaud et Pialoux (1999). 
«Ben je veux que ma fille, elle continue ses études, qu'elle se dise, “j”ai envie de faire ce que je veux". » $\left[\mathrm{M}^{\mathrm{me}}\right.$ Clavien, mère au foyer, mari manutentionnaire, une fille à la scolarité moyenne].

S’ils laissent leurs enfants choisir leur avenir professionnel, signe que le libre choix de s'autodéterminer est une attitude désormais également populaire, comparé aux générations antérieures, (Poullaouec, 2010), il leur arrive parfois d'évoquer un désir ou rêve : les professions les plus souvent citées appartiennent tant aux catégories hétérogènes des fonctionnaires d'État (employé de la poste, infirmière, enseignant) que des professions libérales (médecin, avocat). Les métiers manuels n'ont jamais été cités lorsque les enfants ne se trouvaient pas en échec scolaire à l'école, signe que les familles nourrissent de réelles ambitions scolaires et professionnelles pour leurs enfants ${ }^{8}$.

On ne peut comprendre la place particulière qu'accordent les parents, dans leurs souhaits, au statut de fonctionnaire qu'en regard de leur propre position sociale. Ils espèrent ainsi avant tout que leurs enfants accèdent à une sécurité de l'emploi qui leur fait défaut, leur permettant d'acquérir une respectabilité ainsi qu'une reconnaissance sociale. Si le métier prestigieux de médecin a été souvent cité, comme dans l'enquête de Poullaouec (2010), c'est qu'il permet du point de vue parental d'assurer un «bon salaire »; tout en étant un métier dont on aura toujours besoin, car il est avant tout « utile».

Ainsi, aspirations scolaires et professionnelles souvent élevées allant de pair avec une auto-dévalorisation de sa propre condition sociale témoignent d'une véritable crise de la reproduction au sein des classes populaires, crise qu'on ne peut comprendre qu'en relation avec un système scolaire qui s'est massifié et qui a amené les parents à intérioriser les enjeux scolaires et à intégrer l'école dans leurs stratégies éducatives. Ces aspirations doivent également être mises en lien avec le processus de disqualification d'une condition ouvrière tant au niveau politique (diminution des syndicats ces dernières décennies) qu'au niveau économique. Les ouvriers, qui subissent depuis 1992 en Suisse la précarisation de l'emploi et le chômage, ne veulent désormais plus voir cette condition sociale précaire reproduite. Finalement, le souhait parental de prolongation des études de leurs enfants s'inscrit également dans une dynamique intergénérationnelle de continuité et de reprise des aspirations d'une génération à l'autre (Poullaouec, 2010) ; bon nombre de parents espèrent que leurs enfants réaliseront par la voie scolaire ce qu'ils ont rêvé pour eux-mêmes mais n'ont pu/su se permettre, ayant dû interrompre souvent précocement leur scolarité, faute de pouvoir financer des études longues pour entamer une migration qui les a conduits à occuper en Suisse des métiers tout au bas de l'échelle sociale. Tout

8. De telles ambitions se retrouvent dans d'autres cantons qu'à Genève : Bolzman et al. (2003) soulignent des scolarités plus poussées chez les enfants de parents ouvriers issus de la migration que chez ceux des ouvriers suisses, tant dans le canton romand de Genève que celui alémanique de Bâle ; ils l'expliquent par les espoirs et les attentes que les familles immigrées placent dans le système éducatif. 
porte par ailleurs à croire que cette volonté des parents de voir leurs enfants poursuivre des études dans la voie scolaire au détriment de la filière de l'apprentissage en entreprise constitue un phénomène spécifiquement romand (Meyer, 2009) et plus particulièrement genevois; en effet, ce canton se situe à proximité de la France - pays qui privilégie le modèle des études longues. Le système de formation genevois tend à différencier fortement formation et profession, comparativement à la région alémanique où le système dual continue d'articuler de façon plus étroite pratique au sein de l'entreprise et formation théorique en école. On peut raisonnablement penser que les parents suissesallemands manifestent moins d'enthousiasme quant à la poursuite de leurs enfants dans la voie scolaire, envisageant pour eux plus facilement la voie professionnelle de l'apprentissage (l'auto-exclusion des études étant alors plus massive et probablement davantage "voulue » que «subie » comme " deuxième choix »), ce qui expliquerait alors aussi les choix juvéniles beaucoup plus enclins à se tourner vers la formation professionnelle, mise dans l'opinion publique sur le même pied d'égalité que la formation générale. Il est par ailleurs fort probable que la situation économique en Romandie, et en particulier à Genève, où les taux de chômage sont les plus élevés de Suisse, incite aussi les familles (notamment les étrangers surexposés au chômage) à pousser leurs enfants à poursuivre leur formation scolaire, afin de retarder autant que possible (et avec les meilleurs diplômes) l'entrée dans un monde professionnel caractérisé par sa précarité.

\section{Sentiment d'incompétence ET MOBILISATION SCOLAIRE « MODESTE »}

Désormais plus proches des classes moyennes par leurs «ambitions ", lorsque leurs enfants ne se trouvent pas en grande difficulté scolaire ${ }^{9}$, les familles populaires se différencient de ces dernières par des mobilisations scolaires souvent modestes (et peu en phase avec les exigences des enseignants) mises en œuvre, ainsi que par leurs capacités à orienter les scolarités juvéniles tout au long de la scolarité des jeunes. Évoquant le thème du travail scolaire des élèves à la maison, elles se montrent pourtant aussi très préoccupées (en particulier les mères, qui ont la charge des tâches scolaires), s’investissant à la hauteur de leurs propres ressources dans la scolarité de leurs enfants. Certes, les mères les moins scolarisées manifestent un fort sentiment d'incompétence dans le suivi des devoirs et ne parviennent pas à s'en occuper (voir aussi Montandon, 1991). Mais même dans ce cas, elles n’hésitent pas à déléguer cette tâche aux aînés ou à plus

9. Notre étude montre que, lorsque leurs enfants se trouvent en échec scolaire, les parents révisent plus rapidement à la baisse leurs ambitions scolaires, tandis qu'à situation scolaire juvénile analogue, les parents des classes plus privilégiées tendent à maintenir des ambitions élevées. 
compétent qu'elles (un membre familial, un répétiteur, aux études surveillées) signe qu'elles y accordent de l'importance. Elles se contentent alors d'un contrôle moral du type "t'as fait tes devoirs?»

"[Les devoirs], moi je peux pas contrôler, j'ai pas fait d'études, je comprends pas. Tout ce que je dis, c'est de demander à ses sœurs. "Si t'arrives pas, demande à Lisa ou à Monica de t'aider et c'est tout” ... Par exemple, je lui demande : “T’as fait tes devoirs ?" C'est ça que je lui dis moi, "Fais tes devoirs, après on est tranquille". » $\left[\mathrm{M}^{\mathrm{me}}\right.$ Materazzi, aide-ménagère $]$.

D'autres mères entrent en conflit avec leurs enfants au moment des devoirs, lorsque ces derniers ne comprennent pas leurs explications ou qu'elles ont elles-mêmes des difficultés à les leur expliquer, ce qui amène finalement un certain nombre d'entre elles à se démobiliser des tâches scolaires. Ce type d'attitude, souvent interprété à tort par les enseignants comme un "manque d'intérêt ", doit au contraire être compris comme le signe d'un intérêt «à fleur de peau » pour la scolarité, en milieu populaire. D’autres encore discutent régulièrement à table avec leur enfant sur ce qu'il ou elle a fait en classe durant la journée.

Ce qui frappe à les entendre, c'est l'investissement dont bon nombre d'entre elles font preuve en dépit de leur sentiment d'incompétence, comme si elles persévéraient désormais de plus en plus dans le soutien des devoirs ${ }^{10}$. Le soutien scolaire peut alors être qualifié d'honteux, comme le suggèrent les propos de $\mathrm{M}^{\text {me }}$ Bouzekran, repasseuse : «il y a des devoirs que j'arrive pas à faire... on fait la réponse qu'on a pensé. Après il [parlant de son fils] corrige à la classe "; ou encore ceux de $\mathrm{M}^{\mathrm{me}}$ Merenda, concierge, qui s'excuse devant l'enseignante de son accent lorsqu'elle fait la lecture à son fils, sa langue maternelle étant le portugais : « des fois je lis avec lui, mais je ne lis pas juste ». Ces illustrations témoignent de l'intensification croissante de la préoccupation scolaire en milieu populaire. Ces résultats prolongent ceux trouvés par Montandon (1991) qui soulignait déjà que « la grande majorité des parents sont près de leur enfant pour le surveiller, le contrôler ou l'aider pendant qu'il fait ses devoirs ".

Parler d'un soutien scolaire parental croissant ne signifie pas pour autant qu'il soit en adéquation avec les attentes scolaires des enseignants à l'école primaire, qui adhèrent souvent aux nouvelles pédagogies actives centrées sur le jeu, la créativité, l'autonomie ou encore la réflexion de l'enfant. Il ressort de l'enquête que la mobilisation des parents se base souvent sur des vertus traditionnelles qu'ils ont expérimentées à l'école durant leur propre enfance. Ainsi, l'apprentissage des règles «par cour », sans les discuter reste important.

10. Déchaux (2007) note que la persévérance tend à augmenter en France entre 1992-2003 : l'écart mesuré en temps passé au suivi des devoirs entre celles qui s'avouent très souvent dépassées et celles qui ne le sont que très rarement s'est restreint en dix ans. Voir aussi Héran (1994). 
«Moi je m'énerve parce que j'ai pas appris comme ça, chez nous, l'école jusqu'en 1985 c'était d'une façon, après ça a changé, $X=$ tant, ça fait 12, moi ces méthodes-là j'ai pas connu, pis j'aime pas travailler dans cette méthode, j'aime bien travailler la méthode ancienne disons $15 \times 3=45$. » [M. Mouriño, travailleur dans le bâtiment invalide, qui aime faire faire des tables de multiplication à son fils et qui est décontenancé par les nouvelles méthodes pédagogiques en vigueur à l'école].

Cette préférence pour les méthodes à l'ancienne ne s'explique-t-elle pas aussi par le fait qu'elle est moins exigeante que les techniques nouvelles car elle fait davantage appel à la bonne volonté et au travail qu'à un développement intellectuel, et est donc accessible au plus grand nombre (Perrenoud, 1985) ? De même, il arrive aux parents d'exercer de manière externe un contrôle sur les devoirs des enfants (corrections physiques ${ }^{11}$, promesse d'achats de jouets...).

« [Ā propos de mauvais carnets scolaires] Je lui dis : «tu es nul...qu'est-ce qui t'arrive " ? Quand il m'énerve, je lui donne une tape hein, je le menace, je le frappe. Il a peur, donc il travaille encore mieux [pour l'école]. » [ $\mathrm{M}^{\mathrm{me}}$ Bouzekran].

On se trouve à nouveau éloigné des pédagogies nouvelles défendues par les enseignants, qui valorisent l'autonomie enfantine dans le travail (trouver en soi-même la motivation à se mettre au travail) et de ce fait font peser de fortes attentes sur les élèves, lorsqu'est disqualifié tout apprentissage exclusivement motivé par la crainte des sanctions, l'attrait des récompenses ou le simple conformisme (Perrenoud, 1985).

Notre enquête révèle à quel point les familles populaires romandes ont intériorisé les enjeux scolaires en constituant de plus en plus l'école en objet de préoccupations. Les parents formulent aujourd'hui des ambitions scolaires et professionnelles élevées, tant que leurs enfants ne connaissent pas de difficultés scolaires trop importantes. Déclarant joindre le geste à la parole, ils n'hésitent pas à se mobiliser dans la scolarité de leurs enfants et à suivre les tâches scolaires soit de manière indirecte et distante par délégation à plus compétent que soi, par contrôle moral ou sanction physique en cas de refus juvénile de travailler ou encore en se mobilisant directement et personnellement, en persévérant dans l'aide scolaire malgré leur large sentiment d'incompétence.

On peut dès lors affirmer qu'il n'y a plus désormais d'auto-exclusion a priori de la voie des études en milieu populaire. Les fortes ambitions scolaires parentales sont plus spécifiquement liées au contexte suisse romand, géographiquement proche de la France et qui prend pour modèle les études académiques 
françaises (accès d'une génération au baccalauréat). La différence est grande entre les propos des personnes interviewées et l'enquête ethnographique réalisée dans les années 1920-1950 par Hoggart (1970) à propos de la culture des classes populaires, qui écrivait : "la plupart des membres des classes populaires ne sont pas hantés par des rêves de promotion sociale »; ou encore [à propos du boursier], la famille «ne le pousse pas [scolairement] beaucoup ». La comparaison dans le temps nous permet dès lors de valider la thèse générale de l'intensification de la préoccupation scolaire en milieu populaire, ceci dans le contexte romand.

\section{BIBLIOGRAPHIE}

BEAUD S. et PIALOUX M. (1999) : Retour sur la condition ouvrière. Enquête aux usines Peugeot de Sochaux-Montbéliard. Paris, Fayard.

DECARRO E. (1998) : "L'apprentissage dual à Genève en 1997-1998 », Notes d'information du SRED.

DÉCHAUX J.-H. (2007) : Sociologie de la famille. Paris, Découverte.

DELAY C. (2011) : Les classes populaires à l'école. La rencontre ambivalente entre deux cultures à légitimité inégale. Rennes, PUR.

HÉRAN F. (1994) : "L'aide au travail scolaire : les mères persévèrent " INSEE $\mathrm{n}^{\circ} 350,4$ pages.

HOGGART R. (1970) [1957] : La culture du pauvre. Paris, Minuit.

HUTMACHER W. (1998) : "Démocratisation des études et politique d'accès aux hautes études, Éducateur magazine " Université de Genève, p. 4-8.

KELLERHALS J., WIDMER E. et LÉVY R. (2004) : Mesure et démesure du couple. Cohésion, crises et résilience dans la vie des couples. Paris, Payot.

MONTANDON C. (1991) : L'école dans la vie des familles. Ce qu'en pensent les parents des élèves du primaire genevois. Cahier $n^{\circ} 32$, Genève, SRS.

MEYER T. (2009) : " Can vocationalisation of education go too far? The case of Switzerland ». European journal of vocational Training, 46 (1): 28-40.

MÜLLER C.-A. (2007) : Histoire de la structure, de la forme et de la culture scolaires de l'enseignement obligatoire à Genève au XX siècle (1872-1969), Thèse de doctorat, Université de Genève.

OFFICE FÉDÉRAL DE LA STATISTIQUE (2007) : Mosä̈que de l'éducation en Suisse. Les indicateurs de la formation, OFS, Neuchâtel.

PERRENOUD P. (1985) : "Les pédagogies nouvelles sont-elles élitaires? Réflexions sur les contradictions de l'école active ", SRED, paru dans les actes du colloque Classes populaires et pédagogies, université de Haute-Normandie, Rouen.

POULLAOUEC T. (2010) : Le diplôme, arme des faibles. Les familles ouvrières et l'école. Paris, Dispute.

SAPIN M., SPINI D. et WIDMER E. (2007) : Les parcours de vie. De l'adolescence au grand âge. Lausanne, presses polytechniques et universitaires romandes.

SRED (2011) : L'enseignement à Genève. Indicateurs clés du système genevois d'enseignement et de formation. DIP, Genève. 
